\title{
Calidad de vida y acceso inequitativo al espacio público en Bogotá
}

\author{
José Mario Mayorga Henao \\ Pontificia Universidad Javeriana \\ jmmayorgahenao@gmail.com

\section{Diva M. García García} \\ Pontificia Universidad Javeriana y Centre d'Estudis Demogràfics \\ marcela.garcia033@gmail.com
}

Recibido: noviembre de 2016

Aceptado: diciembre de 2017

Publicado: noviembre de 2018

\section{Resumen}

La inequidad en la distribución de los espacios públicos tiene repercusiones sobre las posibilidades de integración de los grupos sociales coexistentes en el territorio. A partir de esta idea, el artículo explora la relación entre la distribución territorial de la población según su estatus socioeconómico y la accesibilidad al espacio público en Bogotá. Para ello, anteriormente se construyó un indicador de calidad de vida en la escala micro y un indicador de accesibilidad de espacio público que produjo como resultado la cantidad de metros cuadrados a los que accedería una persona desde su vivienda en un recorrido peatonal corto. Se realizó una regresión geográficamente ponderada entre los dos indicadores y se obtuvo como resultado una fuerte relación entre las altas condiciones de vida y la mayor accesibilidad al espacio público, lo cual puso de manifiesto condiciones estructurales de segregación social.

Palabras clave: accesibilidad; espacio público; calidad de vida; indicadores estadísticos; segregación urbana

Resum. Qualitat de vida i desigualtat d'accés a l'espai públic a Bogotà

La manca d'equitat en la distribució dels espais públics repercuteix de manera important en les possibilitats que tenen d'integrar-s'hi els diferents grups socials que hi coexisteixen. A partir d'aquesta idea, l'article pretén explorar les relacions que hi ha entre la distribució territorial de la població segons el seu estatus socioeconòmic i l'accessibilitat al seu entorn. 
Per això abans es va crear un indicador de qualitat de vida a escala micro i un indicador d'accessibilitat d'espai públic que va generar com a resultat la quantitat de metres quadrats als quals podia accedir una persona des del seu habitatge en un recorregut curt a peu. Per relacionar aquestes dues variables es va realitzar una regressió geogràficament ponderada. Com a resultat es va obtenir una forta relació entre els alts nivells de qualitat de vida i una accessibilitat més gran a l'espai públic, la qual cosa va manifestar condicions estructurals de segregació social.

Paraules clau: accessibilitat; espai públic; qualitat de vida; indicadors estadístics; segregació urbana

\section{Résumé. Qualité de vie et inégalité d'accés à l'espace public à Bogotá}

L'inégalité dans la répartition des espaces publics a un impact sur les possibilités d'intégration des groupes sociaux sur le territoire. Á partir de cette idée, l'article explore la relation entre la répartition spatiale de la population selon le statut socio-économique et l'accès à l'espace public à Bogotá. À cette fin, on a élaboré un indicateur de la qualité de vie à l'échelle micro ainsi qu'un indicateur d'accessibilité de l'espace public qui se traduit par la quantité de mètres carrés à laquelle aurait accès une personne depuis son domicile pour un court trajet à pied. Une régression pondérée géographiquement entre ces deux indicateurs a été réalisée, permettant de mettre en évidence une forte relation entre le niveau de vie élevé et une plus grande accessibilité à l'espace public, ce qui montre les conditions structurelles de la ségrégation sociale.

Mots-clés: accessibilité; espace public; qualité de vie; indicateurs statistiques; ségrégation urbaine

\section{Abstract. Quality of life and inequitable access to public space in Bogota}

Inequity in public space distribution has important repercussions on integration opportunities for different social groups coexisting within the territory. Based on this idea, this paper aims to explore the relationship between population territorial distribution according to socioeconomic status and public space accessibility in Bogota, Colombia. To this end, a quality of life indicator was constructed at micro-level scale. In regards to the public space indicator, a matrix between origins and destinations was generated, which produced the amount of square meters of public space a person would access by a short walk from their dwelling. To relate both variables, a geographically weighted regression was performed. A strong relationship was found between the highest levels of quality of life and greater accessibility to public space. This finding reveals the existence of structural conditions which foment social segregation.

Keywords: accessibility; public space; quality of life; statistics indicators; urban segregation

\section{Sumario}
1. Introducción
4. Resultados
2. Aproximación conceptual
5. Conclusiones
$\mathrm{y}$ antecedentes
Referencias bibliográficas

3. Metodología 


\section{Introducción}

El espacio público es un concepto polisémico, analizado desde diversas disciplinas que coinciden en reconocerlo como un bien de uso colectivo cuya función responde a la satisfacción de necesidades urbanas también colectivas. Los aportes del urbanismo a la definición del espacio público se han expresado a través de diferentes modelos de ciudad, siendo en la ciudad tradicional en donde este elemento nació formalmente, con la intencionalidad de constituir un espacio de poder, de encuentro poblacional en el que se expresaran las diferencias y las libertades mediante el uso de la razón (Mayorga, García y Arteaga, 2018).

Sin embargo, a pesar de la amplia producción académica sobre el espacio público, poco se ha explorado sobre su distinta distribución en el entorno urbano, así como las desigualdades en el acceso a los beneficios y a los derechos que genera.

Para el caso de Bogotá ${ }^{1}$ coexisten dos situaciones que complejizan el estudio del tema. Por un lado, numerosas investigaciones han mostrado la distribución desigual de población según el estatus socioeconómico en el territorio (Delaunay y Dureau, 2004; Rodríguez Vignoli, 2008; Aliaga-Linares y ÁlvarezRivadulla, 2010, entre muchos otros). De otra parte, según el Departamento Administrativo Distrital de Espacio Público (2014), para el año 2014 la ciudad proveía 3,93 metros cuadrados por habitante de espacio público efectivo con carácter de permanente, un indicador bajo frente a algunos estándares internacionales que plantean como ideal una relación de 15 metros cuadrados por habitante e incluso frente a la meta propuesta por el Plan Maestro de Espacio Público (Secretaría Distrital de Planeación, 2005), que propone llegar a los 6 metros cuadrados por habitante de espacio público efectivo en 2019.

La coexistencia de una alta segregación residencial y una baja dotación de espacio público lleva a plantear la pregunta principal de este artículo, a saber: ¿Existe en Bogotá un acceso diferenciado a espacios públicos entre los diferentes grupos socioeconómicos concentrados en el territorio?

Dar respuesta a la cuestión anterior implica responder primero a dos interrogantes: ¿ cómo se localizan los grupos socioeconómicos en el territorio de Bogotá? y ¿̨uál es el nivel de accesibilidad al espacio público de la población en la ciudad?

Para responder a dichas preguntas en el presente trabajo se parte de una exploración conceptual y de antecedentes sobre el tema, se procede a la explicación de los métodos utilizados y se presentan los resultados para cada interrogante.

1. Bogotá es la capital y principal ciudad de Colombia. Se emplaza en el centro del país y forma parte del departamento de Cundinamarca. Tiene una extensión aproximada de $33 \mathrm{~km}$ de norte a sur y $16 \mathrm{~km}$ de oriente a occidente. La ciudad cuenta con un área de $1.775,98 \mathrm{~km}^{2}$, de los cuales $1.298,15$ son rurales, 307,36 son urbanos y 170,45 son suburbanos. Según proyecciones del DANE, la ciudad en el 2015 contaba con 7.878 .783 habitantes, de los cuales 7.862.277 estarían ubicados en el área urbana del municipio y 16.506, en el resto (Departamento Administrativo Nacional de Estadística, 2011), lo que representa una densidad aproximada de 4.436,30 habitantes por km2. Se organiza en 20 localidades, que operan como entidades administrativas con objetivo de desconcentrar la población. 


\section{Aproximación conceptual y antecedentes}

\subsection{Espacio público, calidad de vida e inequidad}

$\mathrm{Al}$ demostrarse que el espacio público como atributo físico de la ciudad incide significativamente en la calidad de vida de una población (Krier, 1984; Glazer y Lilla, 1987; Moudon, 1992; Sorkin, 1992; Tibbalds, 1992; Worpole, 1992; Madanipour, 1992; Katz, 1994; Lofti y Koohsari, 2009), su función e importancia han ampliado su rango.

La calidad espacial resulta fundamental en el análisis, tras reconocer la estrecha relación entre calidad de vida y atributos físicos de la ciudad, y se aplica a los espacios públicos por dos de sus principales funciones: la de salud y la de sociabilidad. Por un lado, la disponibilidad de espacio público incide en las condiciones de salud física y mental de los individuos (Jennings y Gaither, 2015), tanto por su provisión de servicios ambientales como por tratarse de lugares donde se llevan a cabo actividades deportivas, recreativas y culturales (Giles-Corti et al., 2005). Adicionalmente, el espacio público incide en la calidad de vida de los individuos por ser un ámbito de sociabilidad informal entre individuos de procedencias sociales diferentes, que requieren de encuentros para facilitar las condiciones de la movilidad social y disminuir los efectos de la estigmatización (Kaztman, 2001)

De acuerdo con lo anterior, cobra mayor importancia la pregunta por la forma en que se distribuyen los espacios públicos en el territorio, teniendo en cuenta que, en palabras de Bourdieu (1999) en él se configuran clasificaciones creadas socialmente, y que evidencian relaciones de poder asimétricas.

En efecto, numerosas investigaciones han mostrado que los espacios públicos, así como otros atributos urbanos, no se encuentran distribuidos de manera homogénea en la ciudad, lo que en muchos casos limita el acceso equitativo entre los distintos grupos e individuos.

Al respecto, Borja y Muxí (2000) muestran cómo la dinámica del mercado inmobiliario, que ha dominado las prácticas de producción material de espacios y de servicios urbanos. A raíz de esta, los espacios públicos pasan a ser elementos residuales de los espacios privados, por lo que su ubicación y diseño se dan de manera posterior a la construcción de los proyectos inmobiliarios. Así, el urbanismo de productos, vinculado a estrategias de competitividad y a una cierta sumisión a la iniciativa privada, ha contribuido a la segregación de agentes sociales en la ciudad (Mayorga y Hernández, 2018). Por todo lo anterior, amplias zonas urbanas presentan fuertes carencias de dotaciones de espacio público, tal como lo han demostrado investigaciones en diferentes ciudades latinoamericanas (Terraza, 2012).

El problema de la distribución de los atributos urbanos positivos entre la población (como lugares deportivos, bibliotecas y espacios públicos, entre otros) ha sido investigado en diferentes contextos, con resultados tan diversos como las áreas y los momentos de estudio. En los Países Bajos, por ejemplo, Van Lenthe et al. (2005) mostraron que no hay diferencias significativas en la proximidad a escenarios deportivos entre los vecindarios de estatus socioeconómicos 
diferentes. En Estados Unidos, por su parte, los resultados han variado con el tiempo. En 1977, Lineberry mostró que en Texas la calidad y la cantidad de servicios urbanos no mostraban una relación positiva con la presencia de anglos ni de personas de alto poder adquisitivo, lo cual explicó a partir de los patrones de urbanización de la ciudad, dado que las zonas más viejas y densas estaban más cerca de los servicios urbanos y que, a su vez, se encontraban más pobladas de pobres y minorías étnicas, ya que las clases superiores estaban migrando a los suburbios. Sin embargo, estudios más recientes en Estados Unidos mostraron que los vecindarios con mayores proporciones de minorías étnicas presentaban las mayores desventajas en términos de acceso a los bienes y a los servicios públicos urbanos (Williams y Collins, 2001).

Para el caso de Hermosillo (México), Lara-Valencia y García-Pérez (2015) evidenciaron que las zonas pobres tenían una participación desproporcionadamente baja en la localización de bienes y servicios públicos, lo que generó una distribución de parques y de espacios abiertos que no solo estaba correlacionada con el estatus socioeconómico, sino que además amplificaba las disparidades existentes.

Según lo anterior, para el contexto latinoamericano, la pregunta por las desigualdades en la distribución de los bienes y servicios públicos de la ciudad tiene una relación directa con el fenómeno de segregación residencial por motivos socioeconómicos ${ }^{2}$, que se extiende en buena parte de las ciudades del subcontinente. Según la CEPAL (2010), las desigualdades que la dinámica del libre mercado crea en las ciudades de todo el mundo se encuentran más acentuadamente en América Latina, debido a la marcada asimetría en la dotación de infraestructuras y de equipamientos sociales (Álvarez, 2013).

En este contexto, la disponibilidad y el acceso a los espacios públicos para todas las capas de la sociedad implica la posibilidad de establecer contactos cara a cara entre clases en condiciones de desigualdad, que, según Kaztman, es uno de los determinantes de la posición de los pobres en las estructuras sociales urbanas de la región. El aislamiento urbano imposibilita que las personas con menos posibilidades económicas puedan acceder a los beneficios de movilización de recursos diferentes a los que circulan en su entorno, lo que reduce la exposición a modelos exitosos de asociación entre esfuerzos y logros y estrecha el conjunto de problemas cuya experiencia y cuyas soluciones puedan compartir con otras clases.

\subsection{Accesibilidad al espacio público}

Para que el espacio público incida positivamente en la calidad de vida de la población, es necesario que, además de estar disponible, sea accesible (Lofti y Koohsari, 2009). Aunque esta última condición no garantiza el uso efectivo,

2. Entendida como la desigual distribución de grupos de población en el territorio, que se manifiesta tanto por la proximidad entre los espacios residenciales de los diferentes grupos como por la homogeneidad social de las zonas en que se organiza una ciudad o por la concentración de ciertos grupos en áreas específicas de la ciudad (Rodríguez y Arriagada, 2004). 
es considerada como un factor funcional relevante en la forma urbana, pues posibilita a los ciudadanos participar de actividades, obtener recursos o beneficiarse de servicios e información (Lynch, 1981).

Para el caso del espacio público, algunas investigaciones han mostrado que existe una asociación entre el acceso objetivo y la percepción subjetiva de los bienes y de los servicios que se utilizan en la ciudad. Dichas investigaciones sugieren que la evaluación positiva que hacen los grupos sociales del espacio público del que disponen en sus barrios está fuertemente asociada a las condiciones objetivas de accesibilidad (Lotfi y Koohsari, 2009).

La importancia de la proximidad física de los espacios de ocio cobra mayor sentido en un contexto de expansión de la ciudad funcional, lo que ha implicado el incremento de las distancias a recorrer para el desempeño de actividades laborales (ante las que hay menor capacidad de decisión), lo que, según diversos autores (Méndez et al., 2009; Gimenez-Nadal y Sevilla-Sanz, 2011), ha producido un resurgimiento de la escala vecinal para la satisfacción de otras necesidades cotidianas. Sin embargo, la proximidad solo despliega sus ventajas cuando la cercanía entre orígenes y destinos se ve complementada con mecanismos de movilidad costeables para la totalidad de la población (Peters et al., 2010). Por ello, la accesibilidad debe ser entendida como una doble condición que combina atributos espaciales y temporales (Marquet y Miralles, 2015).

En este contexto, el modo de transporte peatonal sobresale por sus enormes ventajas: por ser el más eficiente energéticamente (Boer et al., 2007; Delbosc y Currie, 2011), por favorecer las condiciones de salud de quienes lo practican (Koohsari et al., 2013) y por ser el más democrático, pues puede utilizarse sin distingo de ingresos, raza o género (Hanson, 2010).

Adicionalmente, autores como Speck (2012) y Gehl (2014) concuerdan en que caminar es una manera de compartir el espacio público como lugar de socialización y encuentro (Mayorga y Hernández, 2018), por lo que, tal como muestran Valenzuela-Montes y Talavera-García (2015), numerosos estudios (Neutens et al., 2010; Talen y Anselin, 1998, entre otros) han tratado al peatón evaluando la accesibilidad a diferentes lugares en función de la distancia o del tiempo necesario para alcanzarlos, teniendo de fondo una reflexión sobre la equidad en la accesibilidad. Muchas de las investigaciones reseñadas por los autores, además de evaluar la calidad peatonal en el ámbito geográfico respectivo, han reflexionado sobre los factores que propician o no la movilidad peatonal, siendo la distancia de recorrido uno de los elementos más estudiados.

Por los beneficios que presenta la accesibilidad al espacio público y, a la vez, por las restricciones estructurales que puede presentar según las formas de desarrollo urbano, se considera como un atributo que permite hacer examen de las cuestiones de equidad espacial (Talen, 1998).

\section{Metodología}

Metodológicamente se utilizaron tres procedimientos para dar cuenta del objetivo de la investigación: 
a) Calcular un indicador de calidad de vida básico (ICVB) por sectores censales para Bogotá, a partir de un análisis factorial.

b) Calcular un indicador de accesibilidad al espacio público, teniendo en cuenta oferta, demanda e interacción espacial.

c) Establecer la relación estadística y espacial entre el ICVB y el indicador de accesibilidad al espacio público. Dichos procedimientos se describen con detalle a continuación.

\subsection{Cálculo del indicador de calidad de vida (ICV) por sectores censales}

El procedimiento para calcular un indicador de calidad de vida en una escala territorial más adecuada para entender la heterogeneidad de Bogotá partió por espacializar la información del censo del año $2005^{3}$ a escala de sector censal. A partir de la consulta del REDATAM, herramienta dispuesta por el Departamento Administrativo Nacional de Estadística (DANE), se generó una base de datos con las variables disponibles en el censo básico, las cuales fueran conceptualmente útiles para construir un indicador de calidad de vida básico. Esta base de datos atendió a una reflexión conceptual sobre la calidad de vida, incluyendo elementos clave en la medición del fenómeno, agregados en siete dimensiones referidas a factores demográficos, calidad residencial y capital humano.

Las dimensiones y los indicadores se presentan en la tabla 1. En la mayoría de los casos estos indicadores presentaban una escala negativa, ya que cuanto mayor era el indicador, peor la calidad de vida del sector censal. Únicamente el indicador de promedio de años de estudio alcanzado presentaba una escala

Tabla 1. Dimensiones e indicadores o variables a considerar

\begin{tabular}{ll}
\hline Dimensiones & Indicador o variable \\
\hline Hacinamiento no mitigable & Hogares por vivienda. \\
\hline Tamaño del hogar & Promedio de personas por hogar. \\
\hline $\begin{array}{l}\text { Cobertura de servicios } \\
\text { públicos }\end{array}$ & Tasa de hogares sin servicio de acueducto. \\
& $\begin{array}{l}\text { Tasa de hogares sin servicio de alcantarillado. } \\
\text { Tasa de hogares sin servicio de energía. }\end{array}$ \\
\hline Tipología de vivienda & Tasa de hogares en viviendas tipo cuarto. \\
\hline Actividad desarrollada por & Tasa de dependencia económica. \\
\hline los integrantes del hogar & Tasa de población mayor de 17 años sin empleo. \\
\hline $\begin{array}{l}\text { Grado educativo alcanzado } \\
\text { por los integrantes del hogar }\end{array}$ & \begin{tabular}{l} 
Promedio de años de estudio alcanzado. \\
Tasa de población mayor de 17 años sin educación. \\
\hline en la última semana
\end{tabular} \\
\hline
\end{tabular}

Fuente: Mayorga, García y Hernández (2017).

3. Se utiliza el censo del año 2005, dado que es el último disponible en Colombia. 
positiva, por lo cual fue necesario realizar una homologación de la escala de medición de los indicadores (Mayorga, García y Hernández, 2017).

Los indicadores fueron homogeneizados en una escala de 1 a 100 mediante el procedimiento descrito en la ecuación 1. Para homogeneizar las variables se asume un valor máximo y un valor mínimo para cada indicador y se aplica la siguiente ecuación, según sea un indicador de escala negativa o positiva:

Modelo de estandarización de indicadores con escala negativa.

$$
I H_{j}=\left[\frac{I_{j}-\operatorname{Max}(I)}{\operatorname{Max}(I)-\operatorname{Min}(I)}\right] \times 100 \times(-1)
$$

Modelo de estandarización de indicadores con escala positiva.

$$
I H_{j}=\left[\frac{I_{j}-\operatorname{Max}(I)}{\operatorname{Max}(I)-\operatorname{Min}(I)}\right] \times 100
$$

Donde:

$I H j$ es el indicador homegeneizado en el sector $j$.

$I j$ es el valor del indicador en el sector $j$.

$\operatorname{Max}(I)$ es el valor máximo del indicador en todos los sectores.

Min (I) es el valor mínimo del indicador en todos los sectores.

De tal forma, los indicadores de escala negativa y positiva quedan estandarizados, y el incremento del indicador significa mejores condiciones de vida en ambos casos.

Posteriormente se realizó un análisis factorial, por componentes principales, que permitiera producir un índice compuesto que sintetizara el aporte de cada variable. Se entiende el análisis factorial como una técnica multivariada que tiene como objetivo hallar una estructura en una matriz de datos, a partir de la identificación de las correlaciones entre las variables y la definición de factores comunes. Siguiendo a Navarro et al. (2014), la técnica permite analizar la dependencia entre las variables y la asociación entre atributos, lo que permite una revisión de la intensidad de las atracciones y de las repulsiones entre las modalidades que pueden presentar las características cualitativas a partir del estudio de las frecuencias conjuntas observadas.

\subsection{Cálculo de un indicador de accesibilidad al espacio público}

Para abordar el objetivo de describir las diferencias en la accesibilidad al espacio público se adaptó el modelo de recaudación de aportes al espacio público formulado por United Nations Development Programme Chile en el año 2013. En este modelo se hace uso de tres fuentes de datos, a saber: la oferta, la demanda y la red de movilidad en el acceso a espacios públicos (Mayorga, García y Arteaga, 2018). 
En el caso de Bogotá, para calcular la oferta, se utilizaron los datos de espacio público construido, contenidos en el Plan de Ordenamiento Territorial de Bogotá del año 2004, de manera tal que se dispusiera de cercanía temporal con los datos poblacionales. Se utilizaron las plazas, los parques y las plazoletas, espacios de propiedad pública destinados a la utilización común para la satisfacción de necesidades colectivas, de uso libre e indiscriminado. La demanda potencial de acceso al espacio público se calculó a partir de la georreferenciación de la información censal de Bogotá a escala de manzana para el año 2005. Para valorar la relación entre la oferta y la demanda a través de la estimación de costos de tiempo, se diseñó una red peatonal en la que se incluyeron todas las vías de la ciudad.

A partir de la oferta, la demanda y la red, se realizó un análisis para estimar el costo entre orígenes y destinos a partir de una matriz. La matriz fue creada teniendo en cuenta los centroides de los espacios públicos como destinos y los de las manzanas residenciales como orígenes. Teniendo en cuenta que se limitó el alcance de los espacios públicos a una caminata de 10,15 y 20 minutos $^{4}$, se obtuvo como resultado la cantidad de metros cuadrados que alcanzaría una persona desde su vivienda en esos tres rangos de minutos de recorrido a pie.

Se puede describir el procedimiento de la siguiente manera:

1. Se calculó el total de población que tiene acceso a un espacio público, a partir de la sumatoria de la demanda de todas las manzanas que podrían acceder al mismo punto de oferta en 10, 15 o 20 minutos de recorrido caminando.

- Cálculo de población que tiene acceso a un espacio público.

$$
P_{x}=\sum_{i=1}^{n} P_{i}
$$

Donde:

$P x$ es la población total que accede al parque $x$

$P$ es la población.

$i$ es la manzana con acceso inferior a 10,15 o 20 minutos al parque $x$.

2. Se calculó el cociente entre la cantidad de metros cuadrados del punto de oferta evaluado (espacio público) y la cantidad de demanda que puede acceder. Esta parte del procedimiento tiene la siguiente notación:

- Cálculo de superficie de espacio público por habitante (adaptado de United Nations Development Programme Chile 2013).

4. La definición de los rangos de tiempo de caminata para alcanzar un espacio público se hizo a partir de los planteamientos de autores como Speck (2012) y Gehl (2014), quienes establecen que el trayecto para acceder a bienes y servicios urbanos debe estar entre los 10 y los 20 minutos, por lo cual se asume que la caminata media para acceder a un espacio público debe ser de 15 minutos. 


$$
Z_{x}=\frac{m_{x}^{2}}{P_{x}}
$$

Donde:

$Z_{x}$ es la cantidad de $\mathrm{m}^{2}$ por habitante en las manzanas que acceden al parque $x$.

$m_{x}^{2}$ es la superfice del área verde $x$.

$P x$ es la población total que accede al parque $x$.

3. La cantidad de metros cuadrados por habitante correspondiente a cada manzana es la suma de los valores de cada parque que alcanza en 10, 15 o 20 minutos de recorrido. Esta parte del procedimiento tiene la siguiente notación:

- Cálculo de indicador de espacio público por habitante (adaptado de United Nations Development Programme Chile 2013).

$$
\sum_{i=n}^{n} Z_{x i}=Z_{x 1}+Z_{x 2}+Z_{x 3} \ldots+Z_{x n}
$$

Donde:

$$
\sum_{i=n}^{n} Z_{x i} \text { es la cantidad de } \mathrm{m}^{2} \text { por habitante que tiene la manzana } i \text {. }
$$

\subsection{Relación geográfica entre ICV e indicador de espacio público}

Para establecer la relación entre el indicador de ICV y el indicador de accesibilidad a espacio público se utilizó un modelo de regresión geográficamente ponderada (Geographically Weighted Regression, GWR), este último definido como un modelo de regresión local que crea una ecuación para cada elemento del conjunto de datos de la variable dependiente, con la finalidad de capturar las variaciones geográficas (Martínez y Rojas, 2015).

Las diferencias en la utilización de un modelo estadístico global y local como GWR se basan principalmente en la capacidad de este último de ser espacializado y representado geográficamente, lo cual permite estimar las diferencias de la relación de las variables en el espacio. Se trata de un modelo que resulta ventajoso para conocer la heterogeneidad espacial de la relación de las variables (Martínez y Rojas, 2015).

En este modelo se asume que la relación entre las variables varía dependiendo de su localización en el espacio. Adicionalmente, en el modelo GWR se considera que los valores cercanos al punto de análisis tienen un mayor peso para la estimación de los parámetros de regresión. En la asignación de los pesos del modelo GWR se utiliza un esquema de ponderación conocido como Kernel adaptativo (con variación espacial), el cual asigna medidas de variaciones de densidad más grandes, donde estos se encuentran de forma dispersa, y medidas menores donde están más concentrados (Soto, 2013). 
La especificación de los modelos de regresión tradicionales considera que los parámetros son invariantes en el espacio, tal como se puede observar en la tabla 2. En un modelo GWR, en la especificación del modelo se incluyen las coordenadas $(\mathrm{Ui}, \mathrm{Vi})$ y se considera una relación entre el factor y su localización. De tal forma, el modelo toma la siguiente forma (Duque et al., 2011):

— Regresión ponderada geográficamente.

$$
y=\beta_{0}(U i, V i)+\sum_{j}^{k} \beta_{1}(U i, V i) x+e
$$

\section{Resultados}

\subsection{Indicador de calidad de vida básico por sectores censales}

$\mathrm{Al}$ realizar el análisis factorial, se obtuvo como resultado que las diez variables incluidas se resumen a tres factores que logran explicar el $77 \%$ de la varianza, lo cual le da validez al modelo estadístico 5 . Lo anterior se presenta en la tabla 2.

La rotación es un procedimiento estadístico que permite optimizar la distribución de la correlación de las variables al interior de cada factor (figura 1). $\mathrm{Al}$ realizar este procedimiento se obtuvo que el factor 1 explica el 31,4\% de la varianza, el factor 2 explica el $23,9 \%$ de la varianza y el factor 3 el $21,5 \%$ de la varianza. Así, es posible afirmar que se obtuvo un modelo que presenta equilibrio en el peso de sus factores, dado que ninguno duplica el peso de los demás.

Los componentes principales del factor 1 son el ayuno, el promedio de años de educación de la población, el porcentaje de población sin educación y el porcentaje de población desempleada. Teniendo en cuenta que las variables están asociadas a las características de los individuos y su inserción en la estructura económica, se denomina factor socioeconómico.

El factor 2 explica el 23,9\% de la varianza de las variables. Sus componentes principales son el porcentaje de viviendas con energía eléctrica, el porcentaje de viviendas con servicio de acueducto y el porcentaje de viviendas con servicio de alcantarillado. Ya que las variables están asociadas a las características de la prestación de servicios públicos en la vivienda, se denomina factor de servicios públicos en la vivienda.

Por último, el factor 3 explica el $21,5 \%$ de la varianza de las variables. Sus componentes principales son el número de hogares por vivienda, el número de personas por hogar y el indicador de dependencia económica. Estas variables se asocian a la población con respecto a su estructura etárea, la composición del

5. En el análisis factorial, con las variables seleccionadas, se obtuvo un KMO DE 0,837, el cual puede ser considerado alto y permite una adecuación muestral aceptable. Igualmente, se comparó la prueba de esfericidad de Bartlett, con un $P$ valor $(0,00)<0,05$ que rechaza la hipótesis nula y acepta la alterna considerando que el modelo es válido. 
Tabla 2. Matriz de componentes rotados

\begin{tabular}{lccc}
\hline & \multicolumn{3}{c}{ Componente } \\
\cline { 2 - 4 } & $\mathbf{1}$ & $\mathbf{2}$ & $\mathbf{3}$ \\
\hline Personas por hogar & 0,128 & 0,100 & 0,817 \\
Hogares por vivienda & 0,083 & 0,053 & 0,768 \\
Población que ayunó & 0,852 & 0,221 & 0,166 \\
Promedio de años de educación & 0,732 & 0,210 & 0,457 \\
Índice de dependencia económica & 0,607 & 0,218 & 0,663 \\
Porcentaje de población en desempleo & 0,874 & 0,021 & $-0,083$ \\
Porcentaje de población sin educación & 0,782 & 0,267 & 0,431 \\
Porcentaje de viviendas sin energía eléctrica & 0,257 & 0,807 & 0,131 \\
Porcentaje de viviendas sin acueducto & 0,003 & 0,862 & 0,085 \\
Porcentaje de viviendas sin alcantarillado & 0,228 & 0,881 & 0,085 \\
\hline
\end{tabular}

Fuente: Mayorga, García y Hernández (2017).

Figura 1. Gráfico rotado de los componentes

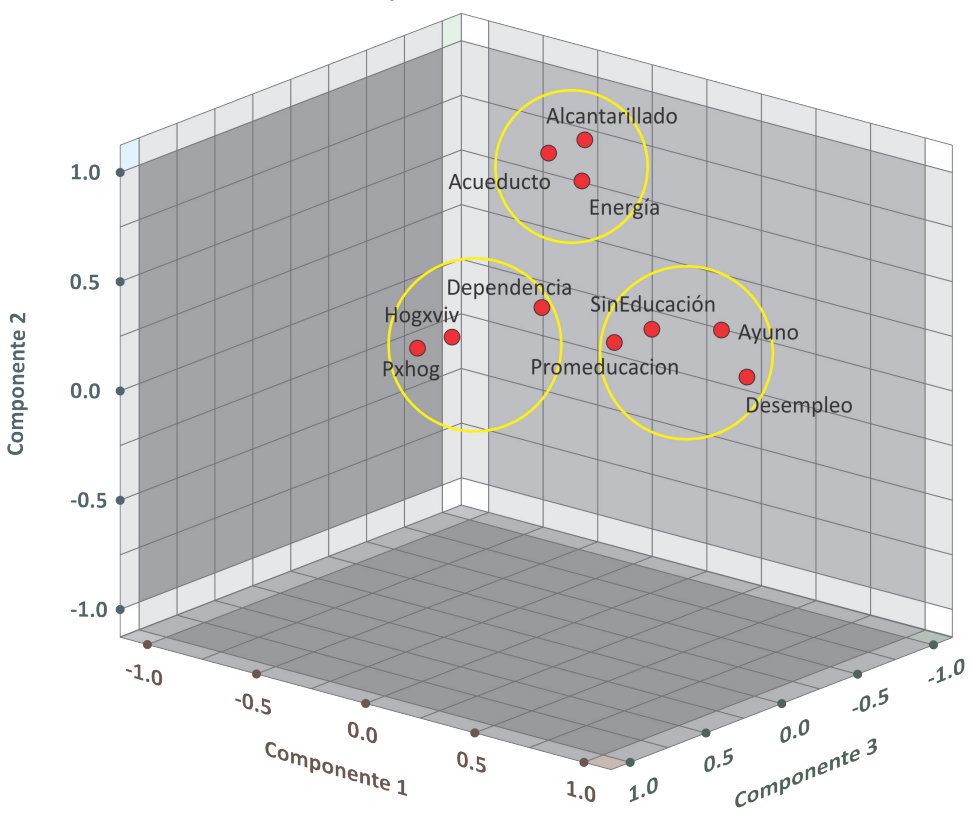

Fuente: elaboración propia.

hogar y la distribución de viviendas en los hogares, por lo cual se denomina factor de composición demográfica (Mayorga, García y Hernández, 2017).

El último procedimiento realizado para obtener un índice compuesto consistió en calcular un promedio simple entre los tres factores, todos con igual peso. En adelante, este índice se denominará ICV básico. 


$$
I C V=\frac{F 1+F 2+F 3}{3}
$$

F1: Características socioeconómicas.

F2: Servicios públicos en la vivienda.

F3: Composición demográfica.

Al espacializar los resultados del ICV básico, se puede observar una tendencia hacia la concentración de los valores altos en dos cuñas que se extienden desde el centro de la ciudad hacia el norte y el occidente y una dispersión de valores bajos en la periferia (figura 2).

Figura 2. Espacialización del ICV básico por sectores censales. Bogotá año 2005

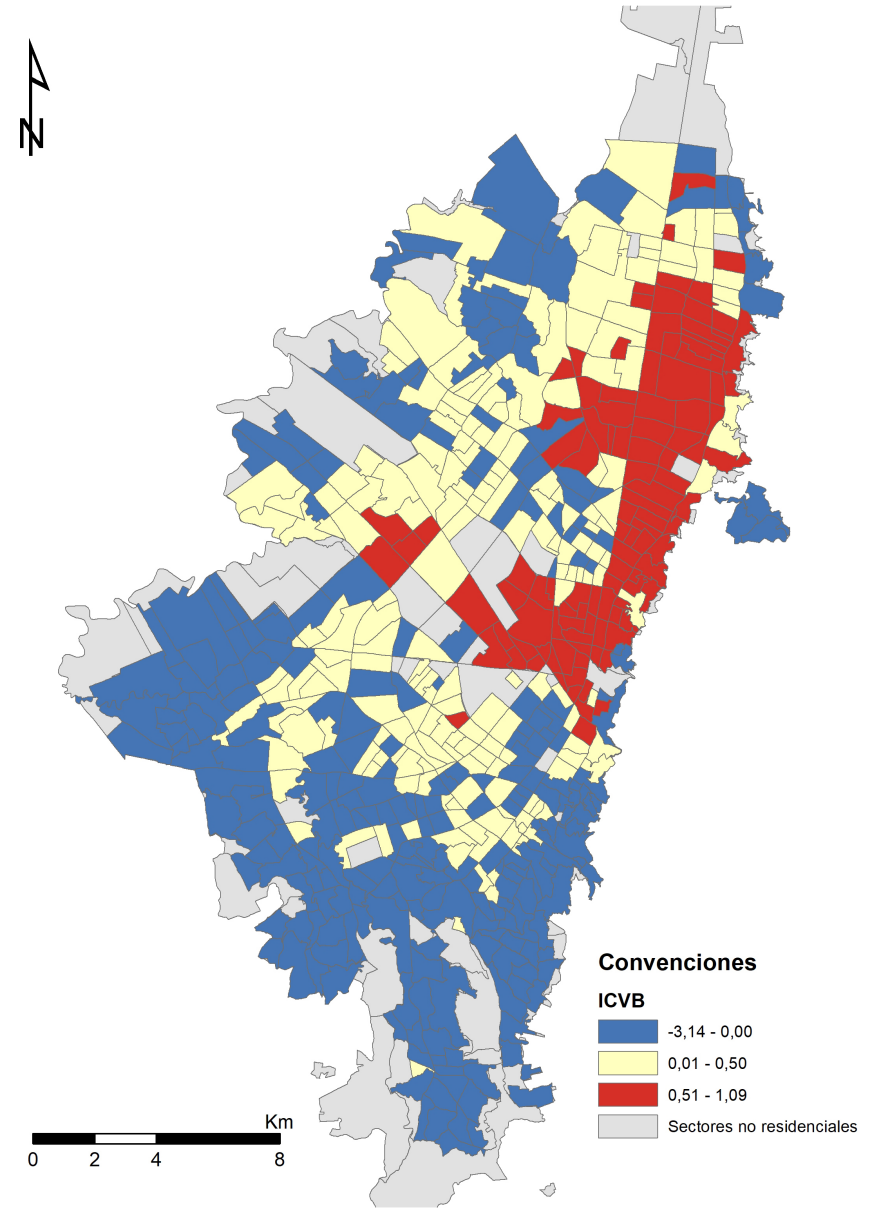

Fuente: Mayorga, García y Hernández (2017). 
Según la espacialización de resultados, se obtiene que más del $50 \%$ de los sectores censales (un total de 262 de los 564 analizados) presentan bajas condiciones de calidad de vida. Dicha situación se concentra en la zona sur de la ciudad, donde se concentra un polo de marcada segregación residencial, que se extiende por las localidades de Rafael Uribe Uribe, Usme, San Cristóbal, Bosa, Ciudad Bolívar y Tunjuelito, en las que se evidencia una alta homogeneidad (cerca del 90\% de sus sectores censales se clasificaron en este rango).

Por su parte, los niveles de vida medios se concentraron en la zona central y en los bordes noroccidentales en 196 sectores censales (34\% del total evaluado). Estos se localizan principalmente en las localidades de Antonio Nariño, Los Mártires, Barrios Unidos, Fontibón, Engativá, Kennedy, Puente Aranda y, en menor proporción, Suba. Estos sectores presentan cercanía con espacios clasificados como de baja calidad de vida, salvo en las zonas más céntricas de la ciudad, donde colindan con las zonas de mejores condiciones de vida.

Estas últimas se encuentran consolidadas hacia el centro y el nororiente de la ciudad, y se presentan en 106 sectores censales, lo que representa el 18\% de las zonas evaluadas. Se localizan en las localidades de Usaquén, Chapinero y Teusaquillo, con una tendencia predominante de condiciones altas concentradas hacia los cerros orientales (Mayorga, García y Hernández, 2017). Adicionalmente, se localizan puntos más dispersos en localidades como Suba, Puente Aranda, Fontibón y Santa fe.

Se observa que, para las zonas de alta calidad de vida, el eje vial de la avenida de Caracas y la Autopista Norte, entre las calles 26 y 170, opera como eje articulador en el que se registra como un comportamiento atípico la presencia de la localidad de Barrios Unidos (al costado occidental del eje entre las calles 63 y 100), en la que predominan los valores medios y bajos.

\subsection{Indicador de espacio público}

Al establecer la distribución de las coberturas de espacio público disponible por habitante en Bogotá a 10, 15 y 20 minutos de caminata, se obtiene una tendencia similar en los tres rangos de tiempo analizados, dado que la mayor cantidad de población de Bogotá se encuentra en los rangos más bajos del indicador, es decir, acceden a menos de 2 metros cuadrados o a entre 2 y 4 metros cuadrados.

A 10 minutos de caminata, el 38\% de la población de Bogotá tiene a su disposición menos de 2 metros cuadrados de espacio público en un rango igual o inferior a 10 minutos de su vivienda, mientras el $35 \%$ cuenta con entre 2 y 4 metros cuadrados en el mismo rango de tiempo y el 14\% cuenta con entre 4 y 6 metros cuadrados. Por otro lado, el 7\% de la población accede a entre 6 y 10 metros cuadrados, y otro $7 \%$ accede a más de 10 metros cuadrados. Es decir que el $87 \%$ de la población de Bogotá accede a menos de 6 metros cuadrados en una caminata de 10 minutos, meta establecida por el Gobierno de la ciudad en su Plan de Ordenamiento del año 2004. 
A 15 minutos de caminata, el indicador presenta la misma tendencia, dado que el $27 \%$ de la población tiene a su disposición menos de 2 metros cuadrados de espacio público en un rango igual o inferior a 15 minutos de su vivienda, mientras el $41 \%$ cuenta con entre 2 y 4 metros cuadrados por habitante en el mismo rango de tiempo y el 16\% cuenta con entre 4 y 6 metros cuadrados. En contraste, el $11 \%$ de la población accede a entre 6 y 10 metros cuadrados y otro $5 \%$ accede a más de 10 metros cuadrados. Es decir que el $84 \%$ de la población de Bogotá accede a menos de 6 metros cuadrados en una caminata de 15 minutos.

A 20 minutos de caminata, el indicador presenta igual tendencia, dado que el $86 \%$ de la población accede a menos de 6 metros cuadrados de espacio público en un rango de 20 minutos a pie de su vivienda.

La figura 3 representa la distribución de la población en los diferentes tiempos y rangos de cobertura señalados y muestra la similitud en la tendencia.

Para hacer una representación espacial de la distribución de las coberturas de espacio público disponible por habitante en Bogotá, se utilizó el indicador a 15 minutos de caminata. Sus resultados se presentan en la figura 4. Se puede observar que los sectores con acceso óptimo a espacio público, los cuales presentan indicadores de más de $6 \mathrm{~m}^{2}$, están en torno a los parques de mayor escala de la ciudad. Sin embargo, en la ciudad hay un predominio de un indicador de acceso a entre 2 y $4 \mathrm{~m}^{2}$. Se concluye, entonces, que hay un desequilibrio entre la distribución de la oferta y la distribución de la demanda y que, por tanto, la ciudad no cuenta con la capacidad de ofrecer el suficiente espacio público para la totalidad de sus ciudadanos.

Lo anterior cobra sentido, ya que no hay una red articulada de espacios públicos que respondan a la distribución de la demanda potencial en Bogotá, lo cual produce que se generen conglomerados de sectores con bajas coberturas de espacio público por habitante, es decir, de menos de $2 \mathrm{~m}^{2}$.

Figura 3. Distribución de población por rango de cobertura

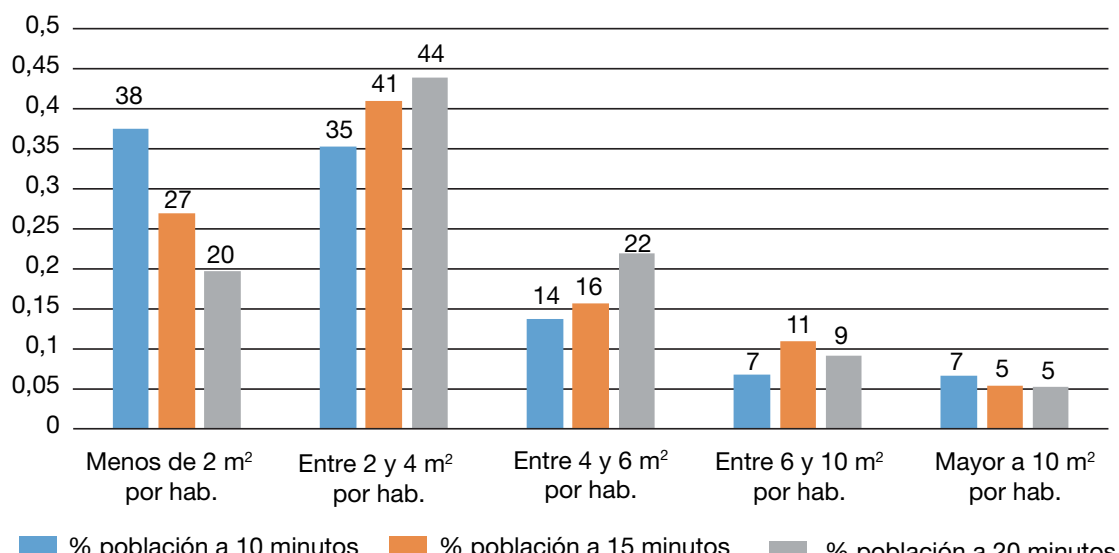

Fuente: elaboración propia. 
Figura 4. Indicador de espacio público de Bogotá

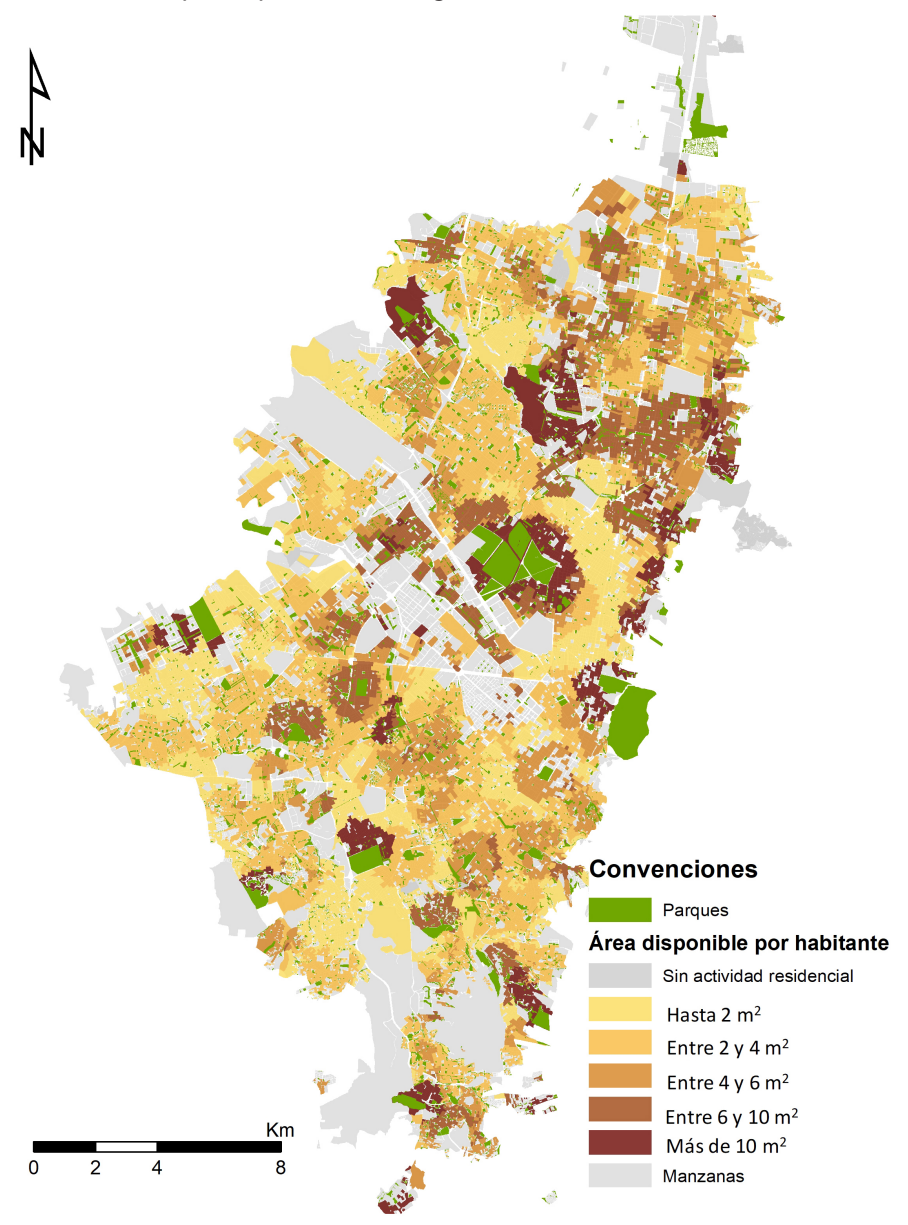

Fuente: elaboración propia.

\subsection{Relación entre ICV e indicador de espacio público}

Al cruzar los indicadores de espacio público por manzana en Bogotá, con la clasificación de sectores según la calidad de vida, se procedió a calcular los indicadores medios según el nivel de calidad de vida.

$\mathrm{Al}$ aplicar la prueba estadística de diferencia de medias se obtiene una diferencia significativa entre los tres niveles ${ }^{6}$ de ICV básico previamente identifi-

6. El ANOVA de un factor muestra que hay una diferencia entre los grupos. Con un $P$ valor de $1,6^{*} 10-8$, hay un nivel de significancia menor a 0,05 y se rechaza la hipótesis nula, aceptando que los promedios por grupo son diferentes. 
Figura 5. Promedio de accesibilidad a espacio público por nivel de ICV

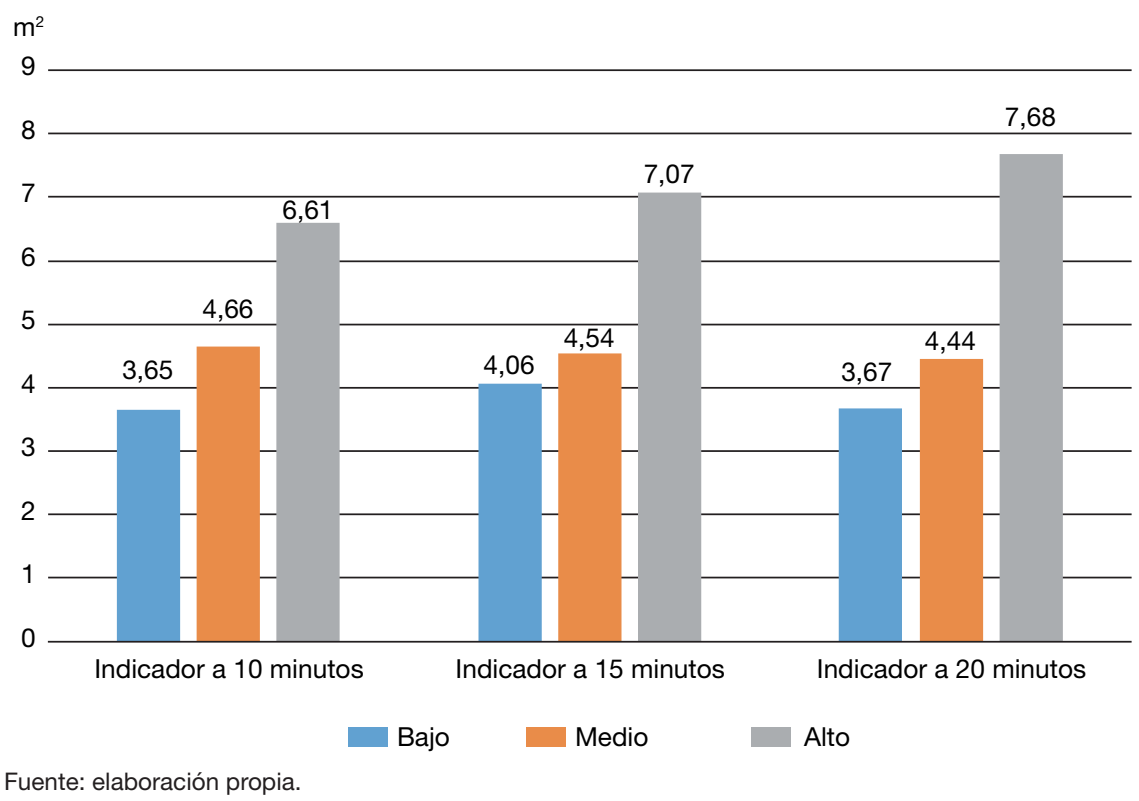

cados. Mientras que los sectores con calidad de vida baja en promedio acceden a 3,65 metros cuadrados en 10 minutos, 4,06 en 15 minutos y 3,67 en 20 minutos, en los sectores con calidad de vida alta el indicador es sensiblemente más alto en los tres rangos de tiempo. En los sectores con calidad de vida media hay un promedio levemente superior al de los sectores con calidad de vida baja, tal como se presenta en la figura 5.

$\mathrm{Al}$ establecer la correlación existente entre el ICV básico y el indicador de espacio público a 10, 15 y 20 minutos, se obtiene que con un $p$ valor de 0,000 están correlacionados en todos los casos. Como se indica en la tabla 3 , el coeficiente de correlación de Pearson indica que el ICVB tiene una correlación de 0,38 con el indicador a 15 minutos, de 0,42 con el indicador a 15 minutos y de 0,33 con el indicador a 20 minutos. Teniendo en cuenta que se acepta estadísticamente la relación entre las variables y que las pruebas gráficas de dispersión (figura 6) indican una tendencia positiva entre las dos, se procede a explorar un modelo de regresión.

Tabla 3. Correlación de Pearson

\begin{tabular}{lcc}
\hline & ICVB & $P$-valor \\
\hline Indicador a 10 minutos & 0,380 & 0,000 \\
Indicador a 15 minutos & 0,400 & 0,000 \\
Indicador a 20 minutos & 0,332 & 0,000 \\
\hline
\end{tabular}

Fuente: elaboración propia. 
Figura 6. Gráfico de dispersión entre las variables

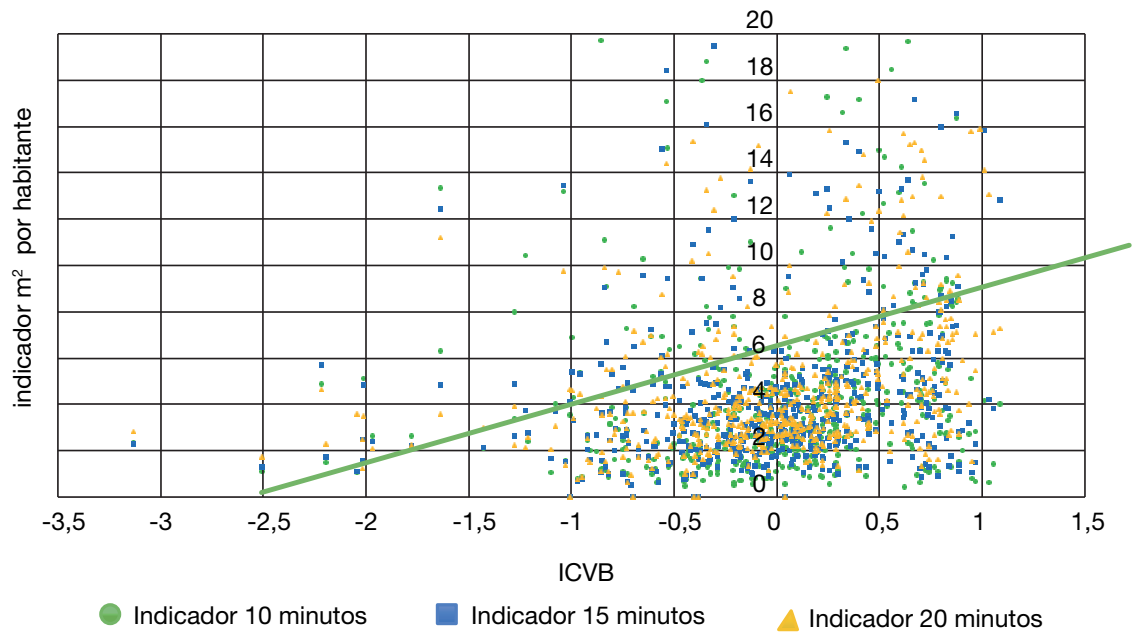

Fuente: elaboración propia.

Ahora bien, teniendo en cuenta que la relación entre accesibilidad a espacio público y distribución del ICV básico en Bogotá es un problema espacial que no se comporta de manera homogénea, se recurrió a un modelo de regresión ponderado geográficamente para establecer una relación local entre las variables. Para ello se utilizó como variable dependiente el ICV básico y como independiente el indicador a 15 minutos, dado que fue el que mayor correlación presentó según el estadístico de Pearson.

Como se aprecia en la tabla 4, el modelo explicativo, ajustado localmente con una relación de 8 vecinos (teniendo en cuenta la contigüidad en bordes y esquina), pudo explicar el $77 \%$ de los valores de la variable dependiente, lo que demuestra que en Bogotá el ICVB se puede predecir espacialmente en función de la accesibilidad al espacio público.

El modelo es válido en tanto los residuales de la regresión, es decir, la diferencia entre los valores pronosticados y los observados, se distribuyen aleatoriamente. La repartición de valores residuales se examinó utilizando la prueba del índice I de Moran. El índice I de Moran determina si el patrón de distribución

Tabla 4. Resultados de la regresión ponderada geográficamente

\begin{tabular}{lcl}
\hline Nombre & Variable & Definición \\
\hline Vecinos & 8 & \\
R2 Ajustado & 0,77 & \\
Variable dependiente & 0 & ICVB \\
Variables independientes & 1 & Indicador 15 minutos \\
\hline
\end{tabular}

Fuente: elaboración propia. 
Figura 7. Ejemplos de sectores
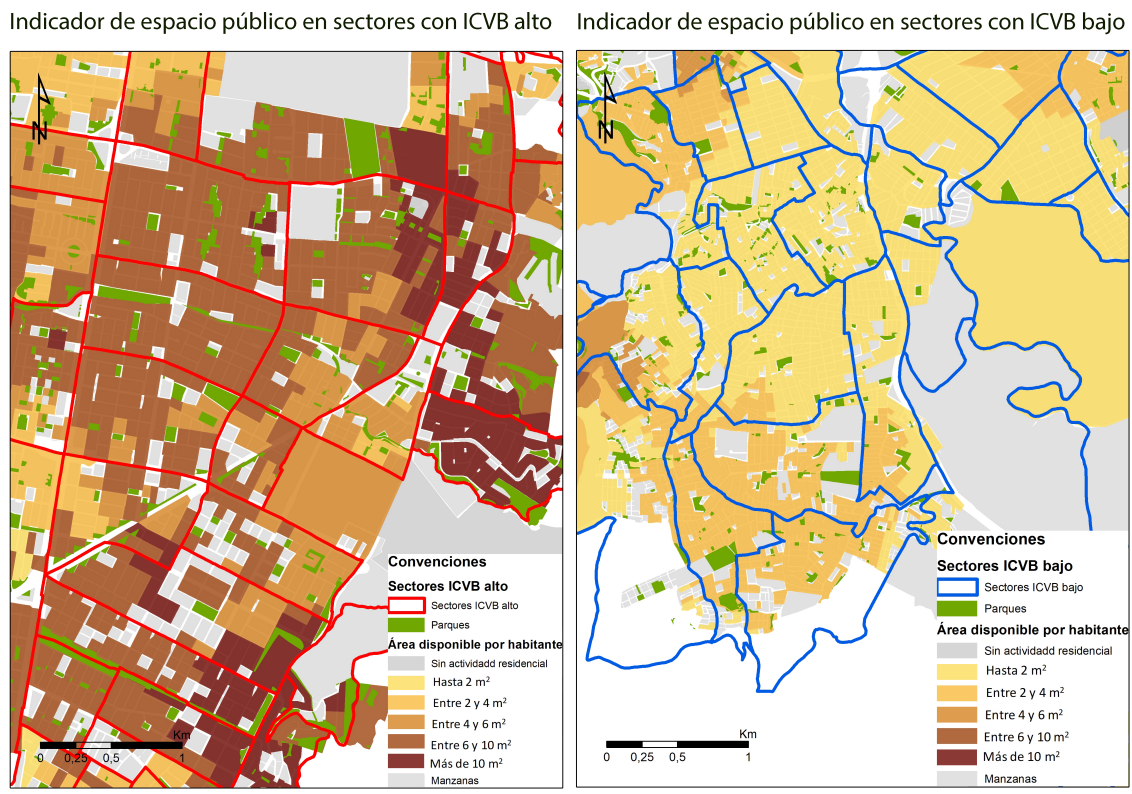

Fuente: elaboración propia.

es agrupado, disperso o aleatorio. En este caso, el modelo generó un $P$ valor de 0,18 , lo que significa que la distribución de residuales es aleatoria. Además, al analizar la cantidad de sectores con un R2 local significativo, se obtiene que es el caso del 53\% del total, lo cual refuerza la validez del modelo.

La relación espacial entre las variables se puede ejemplificar en dos sectores de la ciudad, tal como se ilustra en la figura 7. En el primero, un sector con ICV básico alto al nororiente de la ciudad, se puede observar la existencia de un sistema de espacio público que, sumado a unas bajas densidades poblacionales, permiten que el indicador de accesibilidad al espacio público en 15 minutos de caminata esté principalmente por encima de los $6 \mathrm{~m}^{2}$ por habitante. En el segundo caso, en un sector con ICV básico bajo al suroriente de la ciudad, se puede observar que la dispersión de pequeños espacios públicos no permite garantizar más de 2 metros cuadrados por habitante, dado que se trata de sectores con una alta densidad poblacional y presencia escasa de espacios públicos.

\section{Conclusiones}

La demostrada relación entre niveles de calidad de vida precarios y baja accesibilidad confirma que en el territorio de Bogotá se manifiestan las asimetrías en la dotación de infraestructura y equipamiento social que los informes de CEPAL (2010) revelan como una tendencia en América Latina. 
Lo anterior confirma que la producción del espacio en la ciudad ha respondido a lógicas inmobiliarias privadas que han generado condiciones territoriales y ambientales de injusticia difícilmente reversibles. En este sentido, subraya la necesidad de planear la forma y la estructura urbanas en función de criterios de equidad en el acceso a los servicios de la ciudad, como una forma de garantizar la sostenibilidad de la ciudad como escenario social y ambiental.

Los resultados de este trabajo muestran la urgencia de considerar este tema dentro de las agendas de política pública, al revelar que los pobres tienen significativamente menos posibilidades de acceder al espacio público y que las clases medias presentan un comportamiento solo levemente mejor. Situación que enciende las alarmas a fenómenos como el aislamiento social y una evidente polarización territorial, que provoca que las clases medias compartan las carencias urbanas de los más pobres y, en consecuencia, sus condiciones de exclusión.

De otra parte, en el marco de la reflexión sobre el concepto y las formas de medición de la calidad de vida, es necesario señalar que en este trabajo se tomó como variable un indicador multidimensional que se construyó incluyendo las características familiares y de la vivienda, y, de otra parte, se construyó un indicador de accesibilidad al espacio público, el cual puede ser interpretado como un factor asociado a la calidad de vida en un sentido más amplio, en su relación con la ciudad. Por ello, este tipo de resultados abre la puerta a explorar de manera más profunda y empíricamente la función del territorio en la superación de la pobreza o en su perpetuación, así como la necesidad de plantear la calidad de vida como un concepto más holístico e integral, dentro del que quepan las condiciones de equidad en el acceso a bienes y servicios.

De ahí que resulte de principal interés en esta área de conocimiento la exploración en el contexto colombiano de la relación entre condiciones de salud física y mental y la accesibilidad al espacio público, así como la exploración de la relación entre estatus socioeconómico y el acceso a otro tipo los bienes y servicios públicos en la ciudad, tales como los equipamientos urbanos.

Resultará del mayor interés que estos resultados puedan ser leídos en conjunto o, en contraste, con otros estudios que evalúen el uso efectivo de los espacios públicos por parte de la demanda potencial, ya que una de las limitaciones de la evaluación de la accesibilidad a través del método propuesto es que logra establecer relaciones entre oferta y demanda potencial. Dichos estudios pueden identificar otros elementos, adicionales a la accesibilidad, que incentiven el uso de los espacios públicos, tales como el diseño de los mismos, el mobiliario y otras características de tipo cualitativo.

\section{Referencias bibliográficas}

Álvarez Rojas, Ana María (2013). «(Des) Igualdad socio espacial y justicia espacial: Nociones clave para una lectura crítica de la ciudad». Polis (Santiago), 12 (36), 265-287.

Aliaga-Linares, Lissette y Álvarez-Rivadulla, María José (2010). «Segregación residencial en Bogotá a través del tiempo y diferentes escalas». Documento de Trabajo de Lincoln Institute of Land Policy. Recuperado de <http://bit. ly/19h8zP7>. 
Boer, R.; Zheng, Y.; Overton, A.; Ridgeway, G. K. y Cohen, D. A. (2007). «Neighborhood design and walking trips in ten U.S. metropolitan areas». American Journal of Preventive Medicine, 32 (4), 298-304.

Bourdieu, Pierre (1999). La miseria del mundo. Madrid: Akal.

BORJA, Jordi y MuXí, Zayda (2000). El espacio público, ciudady ciudadanía. Barcelona: Electa.

CEPAL (2010). La hora de la igualdad: Brechas por cerrar, caminos por abrir. Brasilia: Naciones Unidas.

Delaunay, Daniel y Dureau, Françoise (2004). «Componentes sociales y espaciales de la movilidad residencial en Bogotá». Estudios Demográficos y Urbanos, 55, 77-113.

Delbosc, Alexa y CURRIE, Graham (2011). «The spatial context of transport disadvantage, social exclusion and well-being». Journal of Transport Geography, 19 (6), $1130-1137$.

Departamento Administrativo de Espacio Público (DADEP) (2014). Sentido Urbano "Una mirada al espacio público de Bogotá». Alcaldía Mayor de Bogotá. D. C. Bogotá. Recuperado de <https://www.dadep.gov.co/sites/default/files/publicaciones/SENTIDO\%20URBANO3.pdf>.

Departamento Administrativo Nacional de Estadística (DANE) (2011). Proyecciones de población. Recuperado de <https://www.dane.gov.co/index.php/ estadisticas-por-tema/demografia-y-poblacion/proyecciones-de-poblacion>.

DuQUE, Juan Carlos; VELÁSQUEZ, Hamilson y AGUDELO, Jorge (2011). «Infraestructura pública y precios de vivienda: Una aplicación de regresión geográficamente ponderada en el contexto de precios hedónicos». Revista Ecos de Economía, 33 (1), 95-122.

Instituto Distrital de ReCreación y Deporte (IDRD) (2003). Tres parques de Bogotá: Nacional, El Tunal, Simón Bolivar. Bogotá: Alcaldía Mayor de Bogotá.

GEHL, Jan (2014). Ciudades para la gente. Buenos Aires: Editorial Infinito.

GimenEZ-Nadal, José Ignacio y SeVIlla-SAnZ, Almudena (2011). The time-crunch paradox. Netherlands: Springer.

Giles-Corti, Billie; Broomhall, Melissa; Knuiman, Mathew; Collins, Catherine; Douglas, Kate; NG, Kevin; Lange, Andrea y DonOvan, Robert (2005). «Increasing Walking: How Important Is Distance To, Attractiveness, and Size of Public Open Space?». American Journal of Preventive Medicine, 28 (2), 169-176.

Glazer, Nathan y Lilla, Mark (1987). The public face of architecture. Nueva York: Free Press.

HANSON, Susan (2010). "Gender and mobility: New approaches for informing sustainability». Gender, Place \& Culture, 17 (1), 5-23.

HARVEY, David (1996). Justice, nature and the geography of difference. Oxford: Blackwell.

Hillsdon, Melvyn; Panter, Jenna; Foster, Charles y Jones, Andy (2006). «The relationship between access and quality of urban green space with population physical activity». Public Health, 120 (12), 1127-1132.

JenNings, Viniecey y GaITHER, Cassandra (2015). «Approaching Environmental Health Disparities and Green Spaces: An Ecosystem Services Perspective». Int. J. Environ. Res. Public Health, 12 (2), 1952-1968. <https://doi.org/10.3390/ijerph120201952>

Johnston, Ron; Gregory, Derek; Pratt, Geraldiney y Watts, Michael (2000). The dictionary of human geography. Oxford: Blackwell Publishing.

KATZ, Peter (1994). The new urbanism: Toward an architecture of community. Nueva York: McGraw Hill. 
KaZTMAN, Rubén (2001). «Seducidos y abandonados: El aisalmento social de los pobres urbanos». Revista de la CEPAL, 75, 171-189.

KOOHSARI, Mohammad Javad; BADLAND, Hannah y GILES-CORTI, Billie (2013). «(Re) Designing the built environment to support physical activity: bringing public health back into urban design and planning». Cities, 35, 294-298.

KRIER, León (1984). Houses, palaces and cities. Nueva York: St. Martin's Press.

LARA-VAlEnCIA, Francisco y GARCÍA-PÉREZ, Hilda (2015). «Space for equity: Socioeconomic variations in the provision of public parks in Hermosillo, Mexico». Local Environment, 20 (3), 350-368.

LOFTI, Sedigheh y KoOHSARI, Javad (2009). «Analyzing Accessibility Dimension of Urban Quality of Life: Where Urban Designers Face Duality Between Subjective and Objective Reading of Place». Social Indicators Research, 94 (3), 417-435. <https://doi.org/10.1007/s11205-009-9438-5>

LINEBERRY, Robert (1977). Equality and urban policy: The distribution of municipal services. Londres: Sage.

LYNCH, Kevin (1981). Good city form. Cambridge: MIT Press.

MADANIPOUR, Ali (1992). Design of urban space: An inquiry into a socio-spatial process. West Sussex: Wiley.

Marquet, Oriol y Miralles-Guasch, Carme (2015). «The Walkable city and the importance of the proximity environments for Barcelona's everyday mobility». Cities, 42, 258-266.

Mayorga, José Mario; García, Diva Marcela y ArTeagA, Isabel Cristina (2018). «Medición de la cobertura y la accesibilidad al espacio público en Bogotá según el ciclo de vida de los hogares y la estratificación». En: SERRANO, Ronal (ed.). Movilidad Urbana y Espacio Público: Reflexiones, Métodos y Contexto. Bogotá: Unipiloto.

Mayorga, José Mario; García, Diva Marcela y Hernández, Laura (2017). «Calidad de vida y su correlación con los precios del suelo: aproximación a la segregación residencial en Bogotá». Cuadernos de Vivienda y Urbanismo, 10 (19), 22-40. $<$ https://doi.org/10.11144/Javeriana.cvu10-19.cvcp>

Mayorga, José Mario y HernÁndez, Laura (2018). "Medición de la cobertura y la accesibilidad del espacio público en Bogotá, Medellín y Cali». Cuadernos de Vivienda y Urbanismo, 11 (22).

<https://doi.org/10.11144/javeriana.cvu11-22.mcae>

MÉNDEZ, Ricardo (2009). «Procesos recientes en regiones metropolitanas: Transformaciones económicas y reorganización territorial. Algunas interpretaciones y debates actuales». En: V. M. Rodríguez EsPinOsa y J. Bosque SENDra (eds.). La perspectiva geográfica ante los nuevos retos de la sociedad y el medio ambiente en el contexto ibérico. Ponencias del XI Coloquio Ibérico de Geografía. Alcalá de Henares: Servicio de Publicaciones UAH, 37-67.

MARTÍnEZ, Marcela y RoJAS, Carolina (2015). «Regresión geográficamente ponderada para la modelación de la accesibilidad a la red hospitalaria en el área metropolitana de Concepción». Revista Geográfica de Valparaíso, 52, 28-39.

Morar, Tudor; Radoslav, Radu; SPIridon, Luiza y PaCurar, Lu (2014). "Assessing pedestrian Accessibility to green Space using GIS». Transylvanian Review of Administrative Sciences, 42, 116-139.

MORENO JiMÉNEZ, Antonio (2007). «¿Está equitativamente repartida la contaminación sonora urbana?: Una evaluación desde el principio de justicia ambiental en la ciudad de Madrid». Estudios Geográficos, 263, 595-626. 
Morris, Jennifer; Dumble, Paul y Wigan, Ramsay (1979). «Accessibility indicators for transportation planning». Transportation Research A, 13A, 91-109.

Moudon, Anne (1992). «A Catholic approach to organizing what urban designers should know». Journal of Planning Literature, 6 (4), 331-349.

MurILlo, Gabriel y GOMEZ, Victoria (2005). Redefinición del espacio público. Bogotá: Universidad de los Andes. Facultad de Ciencias Sociales. Departamento de Ciencia Política.

Navarro, José; Vanegas, Francisco y Zamora, América (2014). «Migración y desarrollo económico en México: Un análisis factorial de correspondencias». Migración y Desarrollo, 22, 123-145.

Neutens, T.; Schwanen, T.; Witlox, F. y De Maeyer, P. (2010). «Equity of urban service delivery: A comparison of different accessibility measures». Environment and Planning A, 42 (7), 1613-1635. <http://dx.doi.org/10.1068/a4230>

Peters, Peter; Kloppenburg, Sanneke y Wyatt, Sally (2010). «Co-ordinating passages: Understanding the resources needed for everyday mobility». Mobilities, 5 (3), 349-368.

Rodríguez, Jorge y ArriagadA, Camilo (2004). «Segregación Residencial en la Ciudad Latinoamericana». EURE (Santiago), 30 (89), 5-24.

RODRíGUEZ ViGNOLI, Jorge (2008). «Movilidad cotidiana, desigualdad social y segregación residencial en cuatro metrópolis de América Latina». EURE (Santiago), 34 (103), 49-71.

SPECK, Jeff (2012). Walkable City: How downtown can save America, one step at a time. Nueva York: Farrar, Strausadn Giroux.

Secretaría Distrital de Planeación (SDP) (2005). Documento Técnico de Soporte, Plan Maestro de Espacio Público. Bogotá.

SorkIN, Michael (1992). Variations on a theme park: The New American City and the end of public space. Nueva York: Hill and Wang.

Sото, Engelberth (2013). «Regresión ponderada geográficamente para el estudio de la temperatura superficial en Medellín, Colombia». Revista AIDIS de Ingeniería y Ciencias Ambientales, 6 (3), 42-53.

TALEN, Emily (1998). «Visualizing fairness: Equity maps for planners». Journal of the American Planning Association, 64 (1), 22-38.

TALEN, Emily y ANSELIN, Luc (1998). "Assessing spatial equity: An evaluation of measures of accessibility to public playgrounds». Environment and Planning A, $30(4), 595-613$. <https://doi.org/10.1068/a300595>

TERRAZA, Horacio (2012). "¿Las ciudades latinoamericanas poseen suficiente espacio verde público? ¿Qué es suficiente, existe un mínimo?». Ciudades Sostenibles. Banco Interamericano de Desarrollo. Recuperado de <http://blogs.iadb.org/ciudadessostenibles/2012/06/28/la-ciudades-latinoamericanas-poseen-suficiente-espacio-verdepublico-que-es-suficiente-existe-un-minimo/>.

TiBBALDS, Francis (1992). Making people friendly towns: Improving the public environments in towns and cities. Harlow, Essex: Longman Press.

United Nations Development Programme Chile (2013). Propuesta y calibración de mecanismo de recaudación de aportes al espacio público. Recuperado de <http:// www.tendersontime.com/tender/propuesta-y-calibracin-de-mecanismo-de-recaudacin-de-aportes-al-espacio-pblico-38252.php $>$.

VAN LENTHE, Frank; BRUG, Johannes y MACKENBACH, Johan (2005). «Neighbourhood inequalities in physical inactivity: The role of neighbourhood attractiveness, 
proximity to local facilities and safety in the Netherlands». Social Science and Medicine, 60 (4), 763-775.

VAlenZuela-Montes, Luis Miguel y TALAVERA-García, Rubén (2015). «Entornos de movilidad peatonal: Una revisión de enfoques, factores y condicionantes». EURE (Santiago), 41 (123), 5-27.

VÁSQUEZ, Alexis y SALGADO, Marcela (2009). «Desigualdades socioeconómicas y distribución inequitativa de los riesgos ambientales en las comunas de Peñalolén y San Pedro de la Paz: Una perspectiva de justicia ambiental». Revista de Geografía Norte Grande, 43, 95-110.

WORPOlE, Ken (1992). Towns for people: Transforming urban life. Buckingham: Buckingham University Press.

WilliamS, David y Collins, Chiquita (2001). «Racial residential segregation: A fundamental cause of racial disparities in health». Public Health Reports, 116 (5), 404-416. 